\title{
NONLINEAR CONTROL METHOD OF ELECTROMECHANICAL ACTUATOR USING GENETIC ALGORITHM
}

\author{
Huiyan $\mathrm{Li}^{1}$ \\ ${ }^{1}$ Computer School of Hubei Polytechnic University, Hubei 435600, China. \\ Email: lihuiyan hubeiwork@163.com
}

\begin{abstract}
Objective: To study the nonlinear control method of electromechanical actuation system (EMA) based on genetic algorithm (GA) and improve the efficiency of EMA system. Methods: Based on the summary of previous research results and research methods, the nonlinear characteristics of the electric actuator were discussed. Taking the electric actuator as the research object, the nonlinear characteristics that may appear in each part of the electric actuator were comprehensively considered, such as friction and gear gap. According to this analysis, the nonlinear characteristics of each part of the electric actuator were comprehensively studied. The nonlinear characteristics of different parts of the electric actuator were different, and the nonlinear characteristics of different positions were different by Mathlab / Simulink simulation. Results: (1) because the nonlinear characteristics of the gap and friction part have a relatively large impact on the electric actuator, the dead zone of the gap and friction part should not be higher than $2.0 \%$ of the input signal; (2) if there is no dead zone component in the gap, the equivalent stiffness of the transmission structure should not be lower than $1 \mathrm{Nm} / \mathrm{rad}$; (3) it is difficult to trace the nonlinear characteristics caused by friction, so it should be avoided as much as possible. Conclusion: the study provides important research data for the exploration of nonlinear characteristics of electric actuator system.
\end{abstract}

Keywords: Genetic Algorithm; Electromechanical Actuation; Nonlinear Control Method; Electric Actuator.

\section{Introduction}

Electromechanical actuator (EMA) converts the electrical energy into mechanical energy by directly controlling the motor or controlling the movement of load, thus driving various transmission mechanisms to achieve the precise positioning of control objectives.

The EMA is widely used in aviation, industry and agriculture, transportation, and other fields [1].

According to the actuation mode, the EMA system is divided into linear type and rotary type. The former refers to the movement form of system load is linear type, and the latter refers to the movement form of system load is rotary type [2].

Gear reducer is the middle transmission mechanism of the EMA system, which plays an important role in the whole system. The transmission of torque is its main purpose. The nonlinear backlash is essential for transmission. When the minimum backlash space of fixed conditions is met, the gear set can maintain a good working condition, and these conditions have an inevitable relationship with the manufacturing process of the system. However, in the day-to-day work, the gear will produce cause serious wear, resulting in the gradual increase of the gear gap, which makes it more difficult to study the minimum backlash under the minimum fixed condition [3].

As the technology develops and becomes mature, the performance and accuracy of the electric actuator have made rapid progress. In addition, because the management and use of the electric actuator is more convenient, people begin to associate the electric actuator technology with the aerospace industry [4].

Electric actuator technology began to develop gradually in 1950, but at that time, due to the development of technology, the application of electric actuator was only limited to the application in missile steering. At that time, the reasons why the electric actuator could not be applied in the aviation field were that the power of the servo motor in the electric actuator was too small to meet the long-term operation in the aviation field, and the accuracy of the electric actuator was too low to meet the highprecision requirements in the aerospace field [5].

Some scholars used the method of adding inner loop feedback to design the controller, which could compensate the nonlinear characteristics of backlash in the process of electromechanical transmission. Some scholars used the pre-feedback structure in the design of the electric actuator controller, and they used the combination of chaos algorithm and 
conjugate gradient method to calculate the controller parameters [6].

Based on the summary of previous research results and research methods, the nonlinear characteristics of the electric actuator are discussed. The electric actuator is taken as the research object, the nonlinear characteristics that may appear in each part of the electric actuator are analysed.

Moreover, the nonlinear characteristics of each part of the electric actuator are comprehensively studied and compared.

The different nonlinear characteristics of each part of the electric actuator are obtained, and the different nonlinear characteristics of different positions are acquired by Mathlab / Simulink simulation.

\section{Methods}

According to the influence of the nonlinear characteristics, the nonlinear characteristics of the electric actuator system are reclassified.

(1) Dead zone nonlinear characteristics: dead zone nonlinear characteristics in the electrical part, dead zone nonlinear characteristics in the gap part, and dead zone nonlinear characteristics in the friction part;

(2) saturated nonlinear characteristics: saturated nonlinear characteristics in the electrical part;

(3) gap nonlinear characteristics: gap nonlinear characteristics without dead zone components;

(4) friction nonlinear characteristics: nonlinear friction characteristics without dead zone component. Based on the above four non-linear characteristics classification, three kinds of research will be carried out.
First, for the different type and same location, study the impact of different types on the system performance.

Second, for the same type and different location, study the impact of different locations on the system performance.

Third, for the nonlinear characteristics with fixed type and location, study the primary and secondary relations of the influence of the type and position of the system.

The specific non-linear characteristics are classified as follows: the dead zone components in the drive amplifier circuit, the sensitivity limits in the speed and position sensor are dead zone 1, 2, 3 and 4, respectively; the maximum amplitude of the position and speed regulator are saturation 1 and saturation 2, respectively, and the current saturation is saturation 3; the gap between the motor shafts is gap 1; the friction in the motor shaft is friction 1.

\subsection{Nonlinear characteristic modelling}

According to the nature of backlash, the system related to backlash can be divided into three types: input backlash nonlinear system, output backlash nonlinear system, and internal backlash nonlinear system.

In the process of gear movement, the backlash will bring a certain position backlash.

Theoretically, the position change between the driving and driven gears of a group of gears should be linear, but not in the actual use process.

The nonlinear hysteresis of backlash is shown in Eq. 1:

$$
\begin{aligned}
& \theta_{m}(t)=\left\{\begin{array}{l}
m[\theta(t)-\alpha] \\
\theta_{m}\left(t^{-}\right) \\
m[\theta(t)+\alpha]
\end{array}\right. \\
& \dot{\theta}(t)>0 \text { and } \theta_{m}\left(t^{-}\right)=m\left[\theta\left(t^{-}\right)-\alpha\right] \\
& \dot{\theta}(t)<0 \text { and } \theta_{m}\left(t^{-}\right)=m\left[\theta\left(t^{-}\right)+\alpha\right]
\end{aligned}
$$

$\theta(t)$ is the current position of the driving wheel, $\theta_{m}(t)$ is the current position of the driven wheel, $\mathrm{t}$ represents time, $m$ refers to the transmission ratio between the two gears.

In the mechanical and electrical actuation system, the movement of two parts causes the movement of gears, that is, the impact force and mutual extrusion pressure formed by the rotation between the master and slave wheels, which is closely related to the rotation speed and direction between the two gears $[7,8]$.

In the process of establishing the dead zone model, the dead zone model related to gear gap is written as Eq. 2 and Eq. 3:

$$
\tau(t)=\left\{\begin{array}{l}
k[\Delta \theta(t)+\alpha]+c \Delta \dot{\theta}(t) \\
0 \\
k[\Delta \theta(t)-\alpha]+c \Delta \dot{\theta}(t)
\end{array}\right.
$$

$$
\begin{aligned}
& \Delta \theta(t) \leq-\alpha \\
& |\Delta \theta(t)|<\alpha \\
& \Delta \theta(t) \geq \alpha \\
& \Delta \theta=\theta_{m}-\frac{1}{m} * \theta_{d}
\end{aligned}
$$

$\tau$ represents the torque between the two gears, $\mathrm{k}$ and $\mathrm{c}$ are the stiffness and damping coefficient of the gear, respectively, $\theta_{m}$ and $\theta_{d}$ indicate the current position values of the main and driven wheel, 
respectively, $\varpi_{m}$ and $\varpi_{d}$ suggest the current speed value of the main and driven wheel, respectively, and $\mathrm{J}_{\mathrm{d}}$ is the inertia of the driven wheel. $\Delta \theta$ is the current position difference of the master-slave wheel, and 2 times of $\alpha$ equal to the maximum backlash value of the gear.

Figure 1 shows the input and output block diagram of the backlash model.

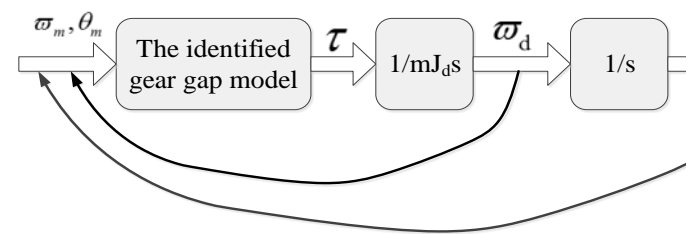

Figure 1: Input and output diagram of backlash model

The Lu Gre friction model can describe the friction behaviour comprehensively and in detail, including the hysteresis and deformation in the friction process. Hence, the nonlinear characteristics of the friction part are discussed through the Lu Gre friction model. The friction nonlinearity described by LuGre model can be regarded as the bristle contact surface, and its expression is shown in Eqs. 4-6:

$$
\begin{aligned}
& \frac{\mathrm{dz}}{d t}=\varpi_{l}-\frac{\left|\varpi_{l}\right|}{g\left(\varpi_{l}\right)} z, \varpi_{l}=\frac{\varpi_{m}}{i} \\
& g\left(\varpi_{l}\right)=F_{c}+\left(F_{s}-F_{c}\right) e^{-\left(\varpi_{l} / \varpi_{s}\right)^{2}} \\
& F=\sigma_{0} z+\sigma_{1} \frac{\mathrm{dz}}{d t}+\sigma_{2} \varpi_{l}
\end{aligned}
$$

$\mathrm{Z}$ is the bristle deformation, $\varpi_{l}$ and $\varpi_{m}$ are the load speed and motor speed, respectively, $i$ is the transmission ratio, $F_{c}$ and $F_{s}$ are the Coulomb friction and the maximum static friction, respectively, $\sigma_{0}, \sigma_{1}$ and $\sigma_{2}$ are the bristle stiffness coefficient, damping coefficient, and viscous friction coefficient, respectively, and $\varpi_{s}$ is the influence factor, which is generally 1 or 2 (in this paper, the influence factor is taken as 1).

Different from the simulation process, there will be a series of additional changes in temperature, humidity, and wear degree in the actual process of friction. Therefore, in the Lu Gre friction model, the variations in friction torque caused by these changes should be added to the model to ensure that the simulation study is consistent with the actual situation. The friction coefficient of the system is the default value when the above changes are not generated normally. If the above changes occur, the friction coefficient needs to be reset. When the parameters in the Lu Gre model change, the friction moment will change, which can be reflected by the friction moment, as shown in Eq. 7.

$$
F=\lambda\left(\sigma_{0} z+\sigma_{1} \frac{d z}{d t}+\sigma_{2} \varpi_{l}\right)
$$

\subsection{Genetic algoithm (GA)}

The core idea of GA is the idea of biological evolution, which regards the problems to be solved as the evolutionary process of organisms. The next generation of population is got through operations like duplication, crossover, and mutation in the next generation of population. The population with low environmental adaptability is eliminated according to the survival method of the fittest, so as to get the most suitable offspring $[9,10]$.

The specific steps of GA are as follows:

First, coding: the process of coding is equivalent to converting the language recognized by human beings into the language recognized by machines, namely converting the target problem into binary form. Second, selection of fitness function: fitness function is the evolutionary standard of a population, and the selection of fitness function has no fixed standard or restriction. After using the corresponding fitness function, different methods are adopted for individuals with different fitness to maintain the evolutionary state of the population. Third, selection algorithm: in order to determine the number of individuals in the next generation and the resulting recombined individuals, the parent individual selects the way in which individuals are passed on to the next generation through selection operation. Fourth, crossover operation: two paired chromosomes exchange with each other and put themselves into local genes to make individual gene change, resulting in new individuals. Fifth, variation: gene changes at a certain site in the chromosome results in gene variation, individual changes, and new individuals.

GA is used to implement the scheme. First, a set of fixed position information is selected as the given position signal $\left\{\theta_{m}\right\}_{i=1}^{n}$. According to the position signal, the corresponding torque sequence $\left\{T_{e}\right\}_{i=1}^{n}$ can be obtained, and then the gear torque $\{\tau\}_{i=1}^{n}$ corresponding to the torque can be obtained. From the approximate dead zone model described above, it can be concluded that the model parameters to be identified are $\{\tau\}_{i=1}^{n} x=[\hat{k}, \hat{\alpha}]$, and the identification error of the model is defined as Eq. 8 here:

$$
e\left(\Delta \theta_{i}, x\right)=\tau\left(\Delta \theta_{i}\right)-\hat{\tau}\left(\Delta \theta_{i}\right)
$$

\section{Results and Discussion \\ 3.1 Identification results of GA}

The parameters used in the identification process of GA are as follows: the actual given stiffness coefficient is 590, and the half value of the maximum backlash is 0.003rad.

The mutation probability $\mathrm{P}_{\mathrm{m}}$ is 0.05 , the crossover probability $\mathrm{P}_{c}$ is 0.85 , the population size is 100 , the maximum number of iterations of evolution is $\mathrm{T}=200$, and the search range of stiffness coefficient and backlash value is: $\mathrm{k}$ is $[500,700]$ and $\alpha$ is [0.002, 0.004]. 
The identification process of GA is as follows: (1) extract the target point in the model, code and record the target point, and regard this data as the initial group; (2) group the random groups and calculate the fitness value of each group; (3) select different operations for different groups, such as mutation and crossover; (4) check whether the evolved algebra meet the expectation. If it meets the expectation, the individual output with the highest degree of adaptation will be achieved; if it does not meet the expectation, continue the operation of step (3) until it reaches the expectation.

According to the identification steps of GA, the model parameters are obtained as follows: the rated value of the identified parameters is $\mathrm{k}=590, \alpha=$ $0.003 \mathrm{rad}$; the identified value using $\mathrm{GA}$ is $\mathrm{k}=$ 595.0021, $\alpha=0.00311$ rad.

\subsection{Simulation results of nonlinear characteristics}

According to the proposed nonlinear research scheme, the following step signal is used as input to simulate and analyse the nonlinear characteristics of saturation, dead zone, gap (excluding dead zone component) and friction (excluding dead zone component).

Take the step signal with the size of $0.1^{\circ}$ as the input, respectively add the dead zone model at the dead zones 1-4, change the size of the dead zone, and get the simulation results as shown in Figures 2-5.

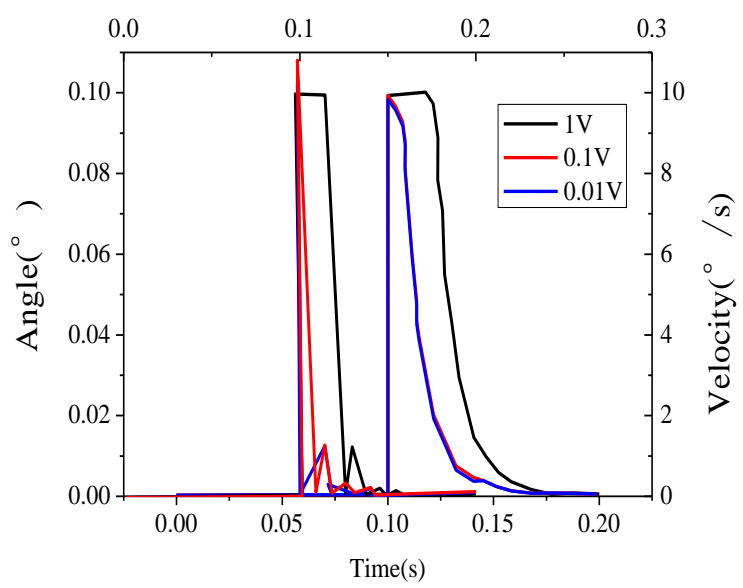

Figure 2: Tracking error of position and velocity when the size of dead zone 1 is $0.01 \mathrm{~V}, 0.1 \mathrm{~V}$ and $1 \mathrm{~V}$

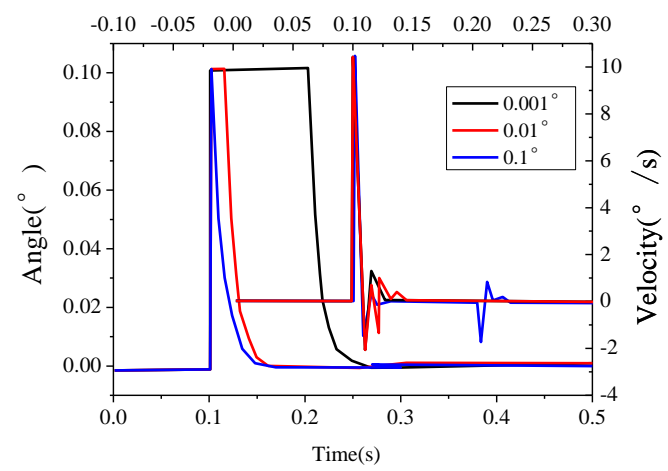

Figure 3: Tracking error of position and velocity when the size of dead zone 2 is $0.001^{\circ}, 0.01^{\circ}$ and $0.1^{\circ}$

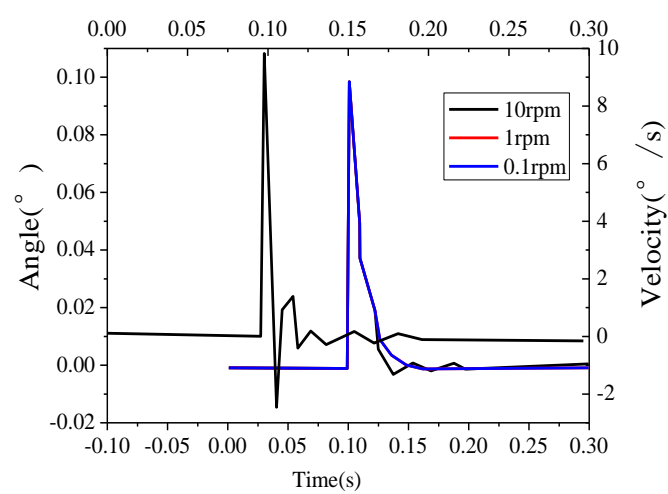

Figure 4: Tracking error of position and velocity when the size of dead zone 3 is $0.1 \mathrm{rpm}, 1 \mathrm{rpm}$ and $10 \mathrm{rpm}$

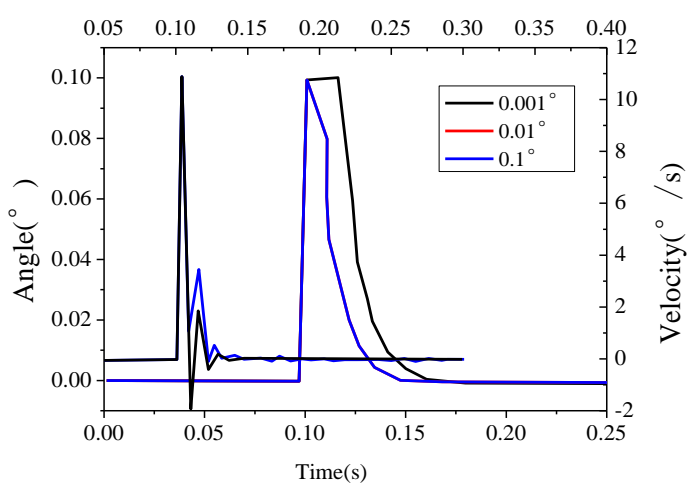

Figure 5: Tracking error of position and velocity when the size of dead zone 4 is $0.001^{\circ}, 0.01^{\circ}$ and $0.1^{\circ}$

Based on the real characteristics of the physical components of the electric actuator system, the nonlinear simulation analysis of the four dead zones is carried out. By combining the simulation results with the practice, it can be concluded that the dead zone component (dead zone 2) in the gap and friction nonlinearity has a significant impact on the tracking performance of the system, which not only causes serious phase lag, but also makes the system oscillate after reaching stability. 
Therefore, the dead zone nonlinearity must be considered in modelling. Set the input signal as a $20^{\circ}$ step signal, cancel the saturation model at saturation
$1-3$, and release the limiting effect. The simulation results are shown in Figures 6-8.

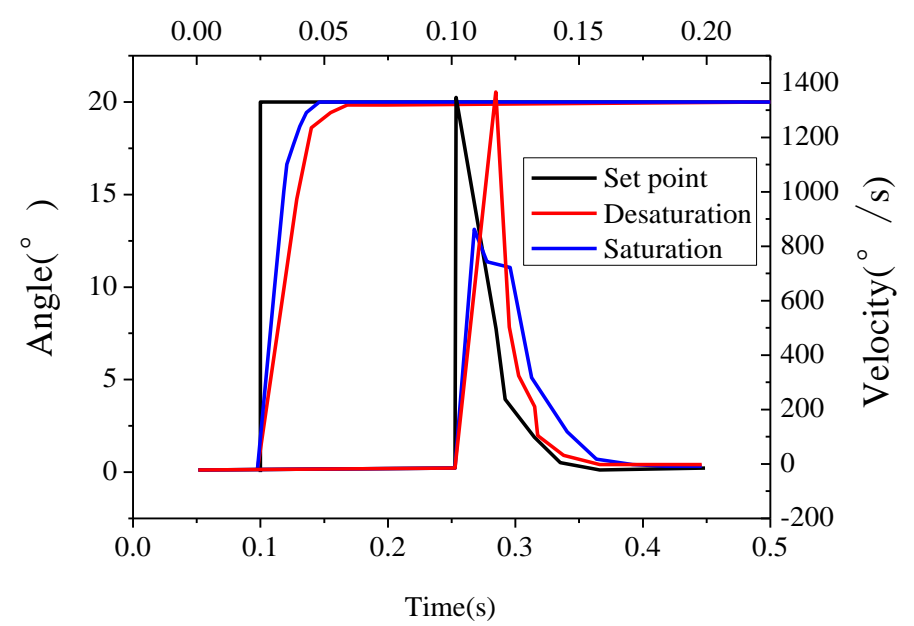

Figure 6: Tracking curve of position and velocity before and after desaturation $1\left( \pm 800^{\circ} / \mathrm{s}\right)$

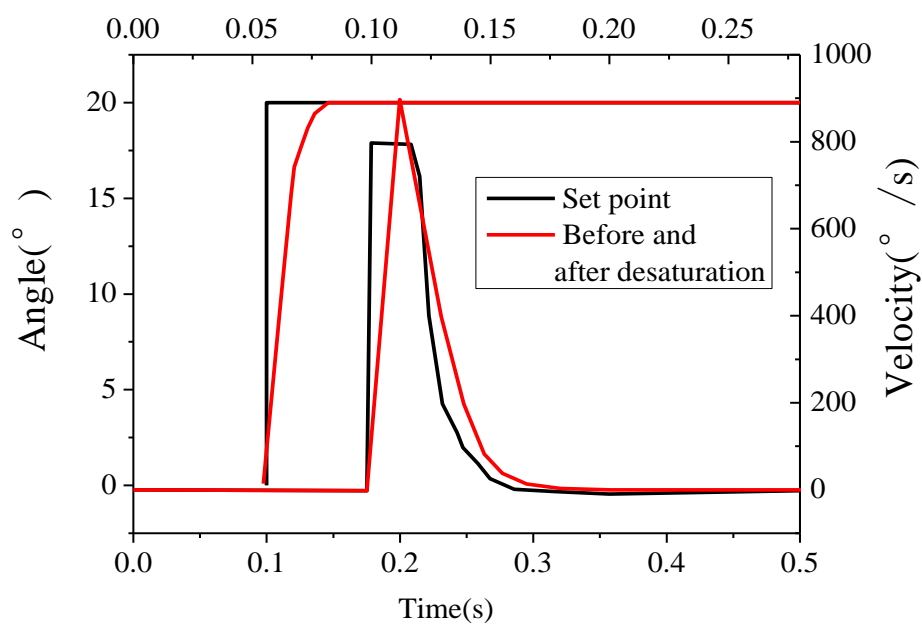

Figure 7: Tracking curve of position and velocity before and after desaturation $2( \pm 40 \mathrm{~V})$

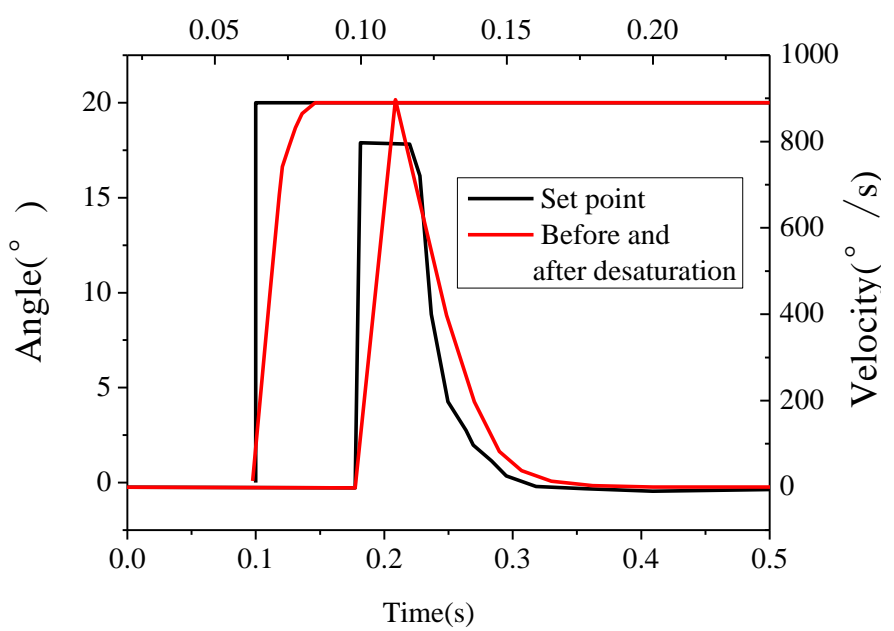

Figure 8: Tracking curve of position and velocity before and after desaturation $3\left( \pm 6.07^{*} 5 \mathrm{~A}\right)$

According to the above results, it is known that the saturation nonlinear characteristics will have a great influence on the life of components of the electric actuator system, so the saturation nonlinear characteristics of the electric actuator system cannot be ignored. Set the input signal as a step signal with a size of $20^{\circ}$, and set the transmission stiffness of $0.01 \mathrm{Nm} / \mathrm{rad}, 0.1 \mathrm{Nm} / \mathrm{rad}, 1 \mathrm{Nm} / \mathrm{rad}$, and $10 \mathrm{Nm} /$ 

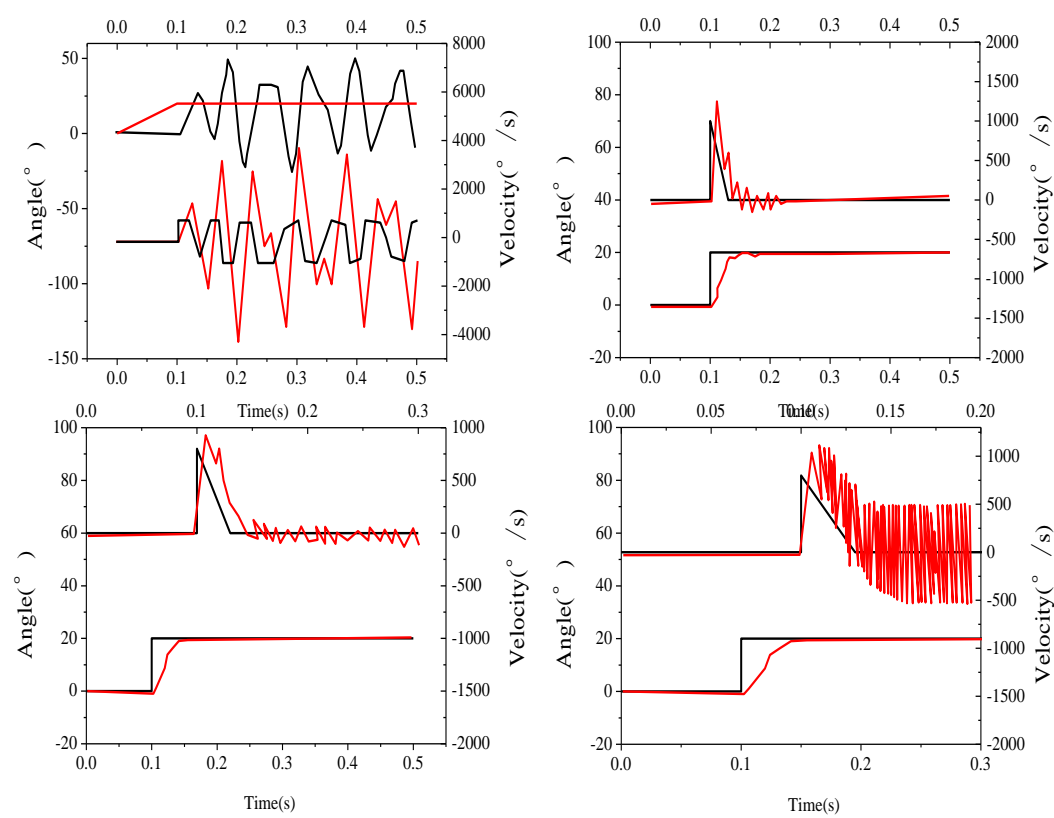

Figure 9: Step response of electric actuator with different gaps

Because the electric actuator is a real object, its physical characteristics are real. In this paper, the non-linear characteristics of the electric actuator without dead zone gap are studied and simulated. It is found that the non-linear characteristics of the gap without dead zone will have a great impact on the stability and accuracy of the system.
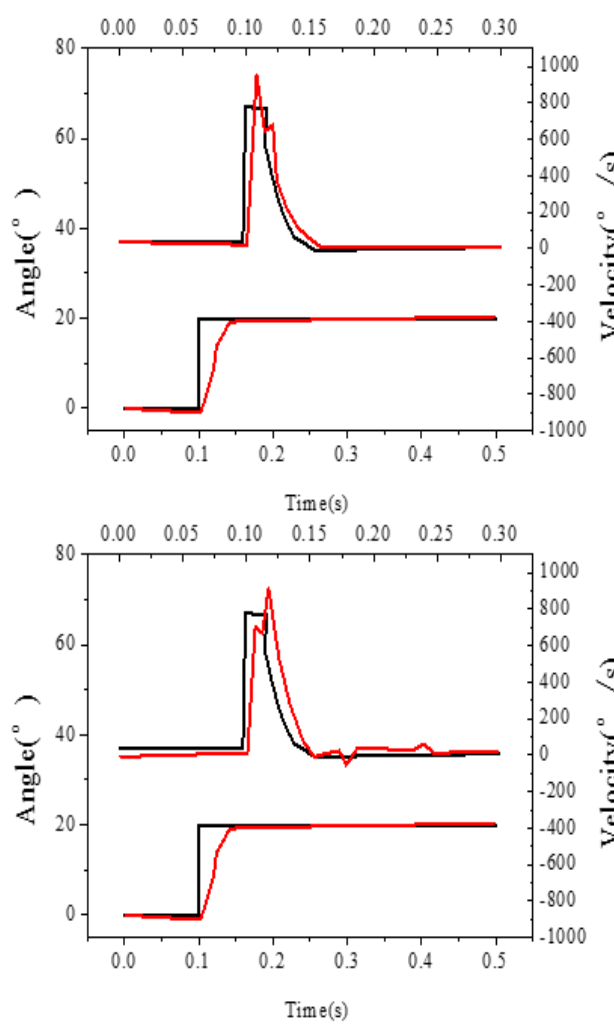

Figure 10: Step response of electric actuator under different friction conditions

When the input signal is a large step signal, with the increase of friction coefficient, the nonlinear characteristics of the electric actuator system will
Therefore, it should be considered in the study of the nonlinear characteristics of the electric actuator.

Set the input signal as a step signal with the size of $20^{\circ}$, and set the friction coefficient of friction part 1 as $0.0001,0.001,0.01$, and 0.1 , respectively.

The simulation results are shown in Figure 10.
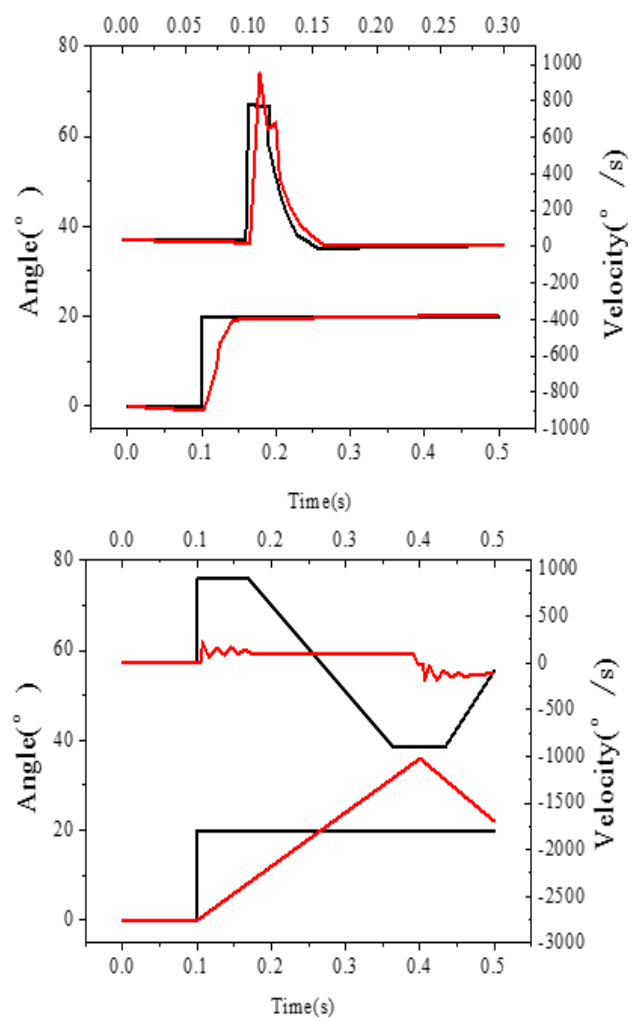

become more and more difficult to track. According to the results, the nonlinear friction characteristics will become the most uncontrollable part when it is 
used with high precision. Therefore, in the study of nonlinear friction characteristics, the nonlinear friction characteristics should be analysed separately and in detail.

\section{Discussion}

Electric actuator technology began to develop gradually in 1950, but at that time, due to the development of technology, the application of electric actuator was only limited to the missile steering. With the development and maturity of the technology, the performance and accuracy of the electric actuator have made rapid progress, and because the management and use of the electric actuator is more convenient, people began to associate the electric actuator technology with the aerospace industry $[11,12]$.

According to the data collected before the research, this paper divides the nonlinear characteristics into four parts: dead zone, saturation, friction, and gap [13]. For the dead zone and gap, dead zone gap model is used, and for the friction part, Lu Gre friction model is used, and different research solutions are proposed. After the research, the following research results are obtained: (1) because the nonlinear characteristics of gap and friction part have a relatively large impact on the electric actuator, the dead zone of gap and friction part should not be higher than $2.0 \%$ of the input signal; (2) if there is no dead zone component in gap, the magnitude of equivalent stiffness of transmission structure should not be lower than $1 \mathrm{Nm} / \mathrm{rad}$; (3) it is difficult to trace the nonlinear characteristics caused by friction, so it should be avoided as much as possible. In this paper, the research of domestic and foreign scholars is analysed, and some of the research results are used for reference.

According to the research purpose of this paper, the research and analysis of nonlinear characteristics of electric actuator based on GA is established, and considerable achievements have been made in this research, but due to the time problem, the research in this paper is not enough, and the research on nonlinear characteristics is not enough.

The following research will be expanded on the basis of this paper, such as the research on the nonlinear characteristics of a certain part.

\section{Conclusions}

With the rapid development of engine technology and engine control technology, the control performance of mechanical and electrical equipment continues to improve, which makes it possible for the electric actuator to be applied to all electric aircraft and high mobility aircraft, and has a broad application prospect. But in this case, the performance requirements of the first electric actuator are gradually increasing. However, the performance of the electric actuator is greatly affected by the nonlinear characteristics of the electric actuator itself.

These nonlinear characteristics cause the negative phenomena such as large error of the electric actuator and slow driving speed, and also cause the flight quality of the aircraft to be greatly reduced. Therefore, the research of nonlinear phenomena is the most essential in discussing electric actuator. It is very important to take necessary measures to reduce or compensate the loss or error caused by these nonlinear characteristics. Hence, on the basis of summarizing the previous research results and research methods, this paper studies the nonlinear characteristics of the electric actuator. Taking the electric actuator as the research object, this paper comprehensively considers the nonlinear characteristics that may appear in each part of the electric actuator, including friction and gear gap, etc. In addition, the nonlinear characteristics of each part of the electric actuator are comprehensively studied.

The research results show that there are a large number of gaps in the transmission part, which will have a serious impact on the electric actuator.

Moreover, most of the adverse effects on the electric actuator are caused by the friction during the operation of the electric actuator, and the other nonlinear characteristics have little impact on the performance of the electric actuator.

\section{References}

[1] J. S. Zhang, H.L. Chen, D. C. Li, "Loss of tension in electromechanical actuation of fiberconstrained viscoelastic dielectric elastomers," EPL (Europhysics Letters), vol.117, no.6, pp. 67004, 2017. 
[2] S. J. A. Koh, C. Keplinger, R. Kaltseis, et al., "Highperformance electromechanical transduction using laterally-constrained dielectric elastomers Part I: Actuation processes," Journal of the Mechanics and Physics of Solids, vol.105, pp. 81-94, 2017.

[3] F. Jian, Jean-Charles Maré, F. Yongling, "Modelling and simulation of flight control electromechanical actuators with special focus on model architecting, multidisciplinary effects and power flows," Chinese Journal of Aeronautics, vol. 30, no.1, pp. 47-65, 2016.

[4] M. S. Loeian, D. A. Ziolkowska, F. Khosravi, et al., "Exfoliated WS2-Nafion Composite based Electromechanical Actuators," Scientific Reports, vol.7, no.1, pp. 14599, 2017.

[5] M. Tanasković, M. Ristanović, S. Stojanović, et al., "Design of electro-mechanical actuator for medium sized helicopter and a test platform for its testing and verification," IOP Conference Series Materials Science and Engineering, vol.393, no.1, pp. 012008, 2018.

[6] Y. B. Meng, Y. T. Zhang, Z. M. Wang, et al., "Couple hysteretic thermo-electro-mechanical performance of piezoelectric actuators for fuel injector," Jilin Daxue Xuebao (Gongxueban)/Journal of Jilin University (Engineering and Technology Edition), vol.48, no.2, pp. 480-485, 2018.

[7] P. Pandiyan, G. Uma, M. Umapathy, "Design and simulation of electro-thermal compliant MEMS logic gates," COMPEL International Journal of Computations and Mathematics in Electrical, vol.37, no.1, pp. 00-00, 2018.
[8] M. Arefi, A. M. Zenkour., "Vibration and bending analysis of a sandwich microbeam with two integrated piezo-magnetic face-sheets," Composite Structures, vol.159, pp.479-490, 2017.

[9] Q. Chao, J. Zhang, B. Xu, et al., "Effects of inclined cylinder ports on gaseous cavitation of highspeed electro-hydrostatic actuator pumps: a numerical study," Engineering Applications of Computational Fluid Mechanics, vol.13, no.1, pp.245-253, 2019.

[10] N. Manikandan, K. Sriram, S. Muruganand, et al., "Stimulation and Analysis of Flexible Bio Polymer Cantilever Based Glucose Sensor," Advanced Science Letters, vol.23, no.3, pp.18751877, 2017.

[11] C. Yang, Z. Liu, C. Chen, et al., "Reduced Graphene Oxide-Containing Smart Hydrogels with Excellent Electro-Response and Mechanical Properties for Soft Actuators," ACS applied materials \& interfaces, vol.9, no.18, pp.15758, 2017.

[12] Y. Yang, N. Schofield, A. Emadi, "Integrated Electromechanical Double-Rotor Compound Hybrid Transmissions for Hybrid Electric Vehicles," EEE Transactions on Vehicular Technology, vol.65, no.6, pp. 4687-4699, 2016.

[13] S. Garlof, T. Fukuda, M. Mecklenburg, et al, "Electro-mechanical piezoresistive properties of three dimensionally interconnected carbon aerogel (Aerographite)-epoxy composites," Composites Science and Technology, vol.134, no.6, pp. 226-233, 2016. 